\title{
A METHODOLOGY TO ACQUIRE AND FORMALISE PROCESS KNOWLEDGE FOR TECHNOLOGY INNOVATION: AN INDUSTRIAL APPLICATION
}

\author{
Caterina Rizzi ${ }^{1}$, Davide Ruozi ${ }^{2}$ and Nicola Gherri ${ }^{2}$ \\ ${ }^{I}$ Dipartimento di Ingegneria Industriale, Università di Bergamo \\ ${ }^{2}$ Dipartimento di Ingegneria Industriale, Università di Parma
}

\begin{abstract}
In this paper, we propose a methodology to acquire and formalize process knowledge for technological innovation and Business Process Re-engineering (BPR). It integrates process modeling and simulation techniques with TRIZ Theory (Russian acronym for Theory of Inventive Problem Solving). The methodology gives the technicians a structured framework, which provides a step-by-step roadmap for BPR through three main phases: acquisition and formalization of AS-IS process knowledge and identification of process criticalities through IDEF techniques, creative problem solving using TRIZ methodology, and modeling and simulation of the new processes, named TO$\mathrm{BE}$, implementing the new technological solutions. The methodology has been experimented for an industrial test case, permitting us to evaluate its efficacy and benefits.
\end{abstract}

Key words: Business process engineering, TRIZ Theory, knowledge management and acquisition.

\section{INTRODUCTION}

The rapidly changing of market imposes continuous improvements of business processes in order to get always-new competitive advantages. An enterprise can take advantage rapidly re-engineering its processes in order to be more flexible and quickly develop new high quality products. In this context, the body of knowledge and its application to solving technical problems is growing more and more (Altshuller 1996), and knowledge 
management is increasingly becoming more and more important in engineering product and process design (Nomaguchi 2001).

While we are trying to solve any kind of problem related to a process or a product we should know how the process or the product is. But process and product are strictly related each other, and in order to optimize a product, we can imagine to refine the process followed to develop or produce it, and to find the knowledge used in the process view. In fact, the knowledge about a product lays inside the product itself and it is generated along the entire process, from design to production. Knowledge comes from a variety of sources, including those within company or outside, and those related to work or not related to work. It is stored as information and data in handbooks, drawings, documents, and electronic means and, in particular, in the head of individuals. Therefore, if we want to capture the knowledge of a product we should study all functions of the business involved in its life cycle. A business process comprehends different kinds of knowledge: strategic knowledge, structural knowledge, systems knowledge, cultural knowledge and routines and embodied knowledge (Scarbrough 1996). Every single brick captured from these levels of knowledge builds the product we analyze; therefore, it is strategic to formalize this information following the product life-cycle through the business process. Modeling the process focusing the attention on the product can be a way to capture and formalize all information at different levels of detail, from the knowledge embedded within the structure of the organization to the tacit knowledge embedded in routines and embodied in individual employees. It is possible to describe the different levels of knowledge involved in each task through a multilevel process modeling. Different techniques can be used to achieve this aim, but it is extremely important to select proper tools and define a road map to guide the process analyst.

Another important issue, when dealing with product or process problem solving, is metrics: we must define and measure what we mean by improvement by (Mayer ---), considering techniques and tools that permit to measure and report on productivity and innovation. Thus there is the need to define methodologies and develop proper tools not only to help scientists and technicians in finding new ideas and solutions faster and faster without loosing among different sources of knowledge, but also to quantitatively measure new technological solutions.

In this context, our objective has been to define and experiment a methodology to acquire and formalize business process knowledge for technological innovation and Business Process Re-engineering (BPR). In the following we describe developed methodology and its application to an industrial test case. 


\section{METHODOLOGY DESCRIPTION}

The proposed methodology integrates process modeling and simulation techniques with a commercial semantic processing engine, specifically the TechOptimizer by Invention Machine Corp. According to the definition given by (Mayer ---), our methodology gives the technicians a structured framework, which provides a step-by-step roadmap, techniques and tools for technological innovation and BPR.

The methodology, schematized in Figure 1, requires three main activities:

- Acquisition and formalization of AS-IS process knowledge (Bozza 1998) and identification of process criticalities using IDEF techniques (http://www.idef.com);

- Creative problem solving using TRIZ (Russian acronym for Theory of Inventive Problem Solving) Theory (Altshuller 1996) with the support of TechOptimizer package (http://www.invention-machine.com);

- Modeling and simulation of the new processes, named TO-BE, implementing the new technological solutions (identified in the previous step) as well as for the AS-IS process.

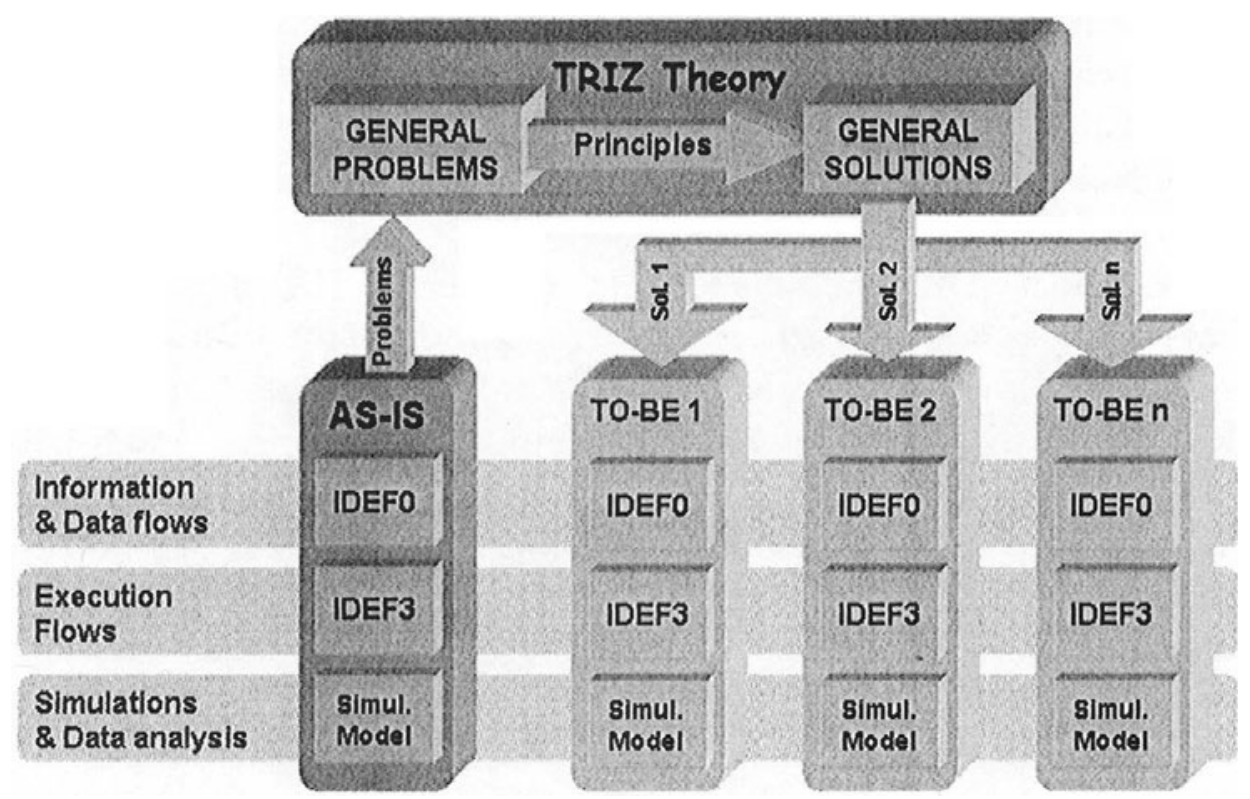

Figure 1. Proposed Methodology

The first phase consists in studying the AS-IS process, i.e., the process currently carried out at the company, collecting process knowledge through interviews with the experts of the process. During this activity the knowledge regarding both the product and the process, usually spread 
among the technicians and company departments, is acquired and formalized.

The AS-IS model permits to highlight process problems and area of possible improvements, and represents the term of comparison to evaluate quantitatively the effectiveness of innovative solutions. In literature, we can find different techniques to represent process knowledge: ARIS, IDEF, UML, etc.

Studies on business modeling came to the conclusions that there isn't a universal tool; the challenge is to find the right tool (http://www.cit.gu.edu$\mathrm{au} / \sim$ noran/cit_6114). Thanks to our experience carried out in several industrial contexts on product-development processes reengineering, we adopted IDEF techniques. We consider IDEF0 for information and data flows representation and IDEF3 for execution flows. They permit to represent process knowledge with a graphic language, simple and easy to be understood and used also by people without a technical background. This facilitates the communication among work teams with different competences, particularly important to validate the process model with process experts and to ensure that collected information (process and/or product knowledge) has been correctly formalized.

Typically following steps are performed:

- Capture process/product knowledge through interviews with company experts, and technical documentation;

- Graphical represent and formalize the process knowledge through IDEF0 technique.

In particular, IDEF0 permits to define the functional model of the process that includes a structured representation, at different level of details, of functions/activities, and what is needed to perform those functions. It also permits to identify what the current system does right and what the current system does wrong.

- Review the IDEF0 model with the process experts and consolidate the model that correctly photographs the current status of the process. This step is important because it permits to define a common view of the process agreed by all persons (technicians and not) involved in the process. The resulting final model represents the starting point for the following phases and for comparing results.

- Define the IDEF3 model that shows the process execution flow. IDEF3 captures precedence's and causality relations between situations and events (sequential activities/sub-processes, concurrent activities/subprocesses, alternative activities/sub-processes) by providing a structured method for expressing knowledge about how a system, process, or organization works. Therefore, it permits to identify and formalize decisional processes embedded within the business process itself. 
- Collect data about execution times and probability that production mistakes can occur leading to a partial or total re-execution of activities/sub-processes.

- Define and execute simulation model to gather quantified sample data that will be used to evaluate and compare the proposals for the reengineered process.

Summarizing, at the end of this phase, the outcomes are: acquisition and formalization of process knowledge, formalization of decisional processes, and identification of process criticalities.

Second phase consists in analyzing and solving problems identified in the previous one using TRIZ methodology. TRIZ underlying idea is that invention has logical rules and principles that lead from problem to solution (Altshuller 1996), i.e., there are common patterns (called Inventive Principles) in ways of solving problems that, extracted and coded, technologists and researchers can use to obtain the capability to solve problems creatively. Summarizing, the basic axiom of TRIZ is: objective laws govern the evolution of any technical system. TRIZ has built a system of abstract principles and laws together with a huge collection of facts and applications examples in a readably applicable manner (Nakagawa 1999).

Figure 1 and 2 show in a simply way TRIZ methodology to solve problems: (1) once acquired problem specifications, (2) the problem is first generalized and formalized defining a structural-functional model, (3) analyzing world of science and technology, general solutions are found, (4) then these solutions are specialized for the specific problem.

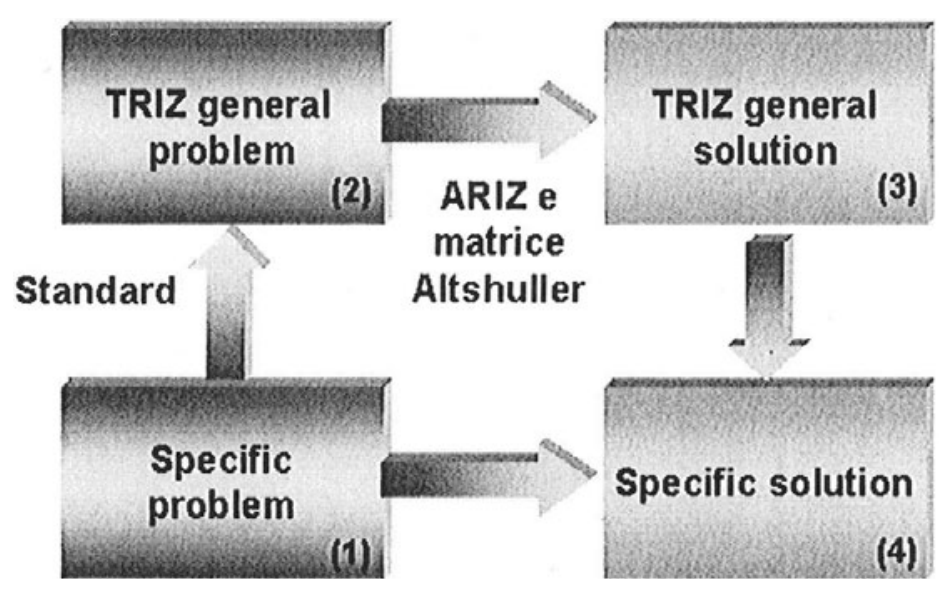

Figure 2. TRIZ Theory

This phase is carried out using TechOptimizer package (Invention Machine Corp.) that implements the TRIZ knowledge bases and tools (e.g., ARIZ algorithm) integrated with AI technologies, incorporating value 
analysis, cost analysis, and function analysis to effectively solve manufacturing problems and quantitatively evaluate technical solutions.

TechOptimizer makes available to the designers user-friendly tools for knowledge management and extraction (Lawton 2001; Altshuller 2000) exploring alternative designs, and finding innovative technical solutions.

Also for this phase, it is possible to define a sequence of typical steps to be performed:

- On the basis of AS-IS model and identified criticalities, define the structural-function model of the product and/or of the process highlighting components and/or operations (functions) where the problems are, using for both models a graphic language. This means to specify a model of the problem/s.

- Apply TRIZ strategies by means of TechOptimizer solution tools to extract innovative technical solutions. The selection of solving strategies, and therefore solution tools, depends on problem statement and technicians' objectives.

- Analyze identified technical solutions and apply them to the specific problem.

Following the same steps performed for the AS-IS process, last activity consists in modeling the new processes (TO-BE processes) that implement the new technical solutions. Main objective of this phase is to permit the process experts to highlight advances and changes with respect to the AS-IS process and to quantitatively evaluate the new scenarios defined according to the "innovative solutions" identified by means of TRIZ theory.

\section{THE CASE STUDY}

The described methodology has been experimented within an industrial context. The study case refers to the production of a mixer component, named cowless (Figure 3).

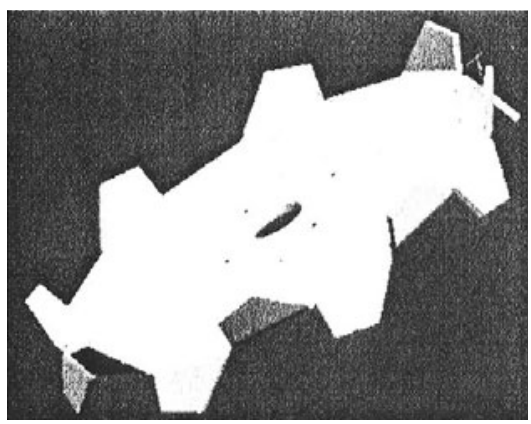

Figure 3. A typology of cowless 
They are produced in several types with different teeth, dimensions and holes. Because of products variety, the actual process is low automated and consequently it takes a lot of time, is labor-intensive and expensive, so most of cowless production was outsourced. The involved company is a SME and its need was to re-engineer the process in order to improve process efficiency, optimize resources, guarantee product quality and get production process knowledge.

Therefore main objectives have been:

From academic point-of-view:

- Experiment the proposed methodology to acquire and formalize knowledge for technical innovation;

- Recognize and evaluate methodology benefits.

From company point-of-view:

- Re-engineer the production process of the mixer component;

- Acquire process knowledge and define a new flexible production process;

- Reduce production time and resources in order to produce cowless inside the company.

In the following, we describe activities performed according to the proposed BPR methodology: acquisition and formalization of AS-IS process knowledge, identification of innovative solutions, and, finally, definition and evaluation of the TO-BE processes.

\subsection{Acquisition and formalization of the AS-IS process knowledge}

We proceeded following the procedure described in Section 2. First steps consisted in studying the AS-IS process considering either the inside production process or the outsourced one. Therefore, two AS-IS models have been realized. Then, simulations have been executed to get sample data on meaningful aspects of both processes (internal and outsourced): production time and manpower. We identified process criticalities and possible area of improvements as follows: re-engineer the shearing operation (Figure 4) adopting more flexible machinery and modify bending operation to reduce dead times for machine set-up. In fact, the last operation was performed in two steps (Figure 5): the first set of teeth with the same slope was bended one by one, then punch and die were turned and the second set of teeth was bended. Figure 4 and 5 show the IDEF0 diagrams describing mentioned operations. 


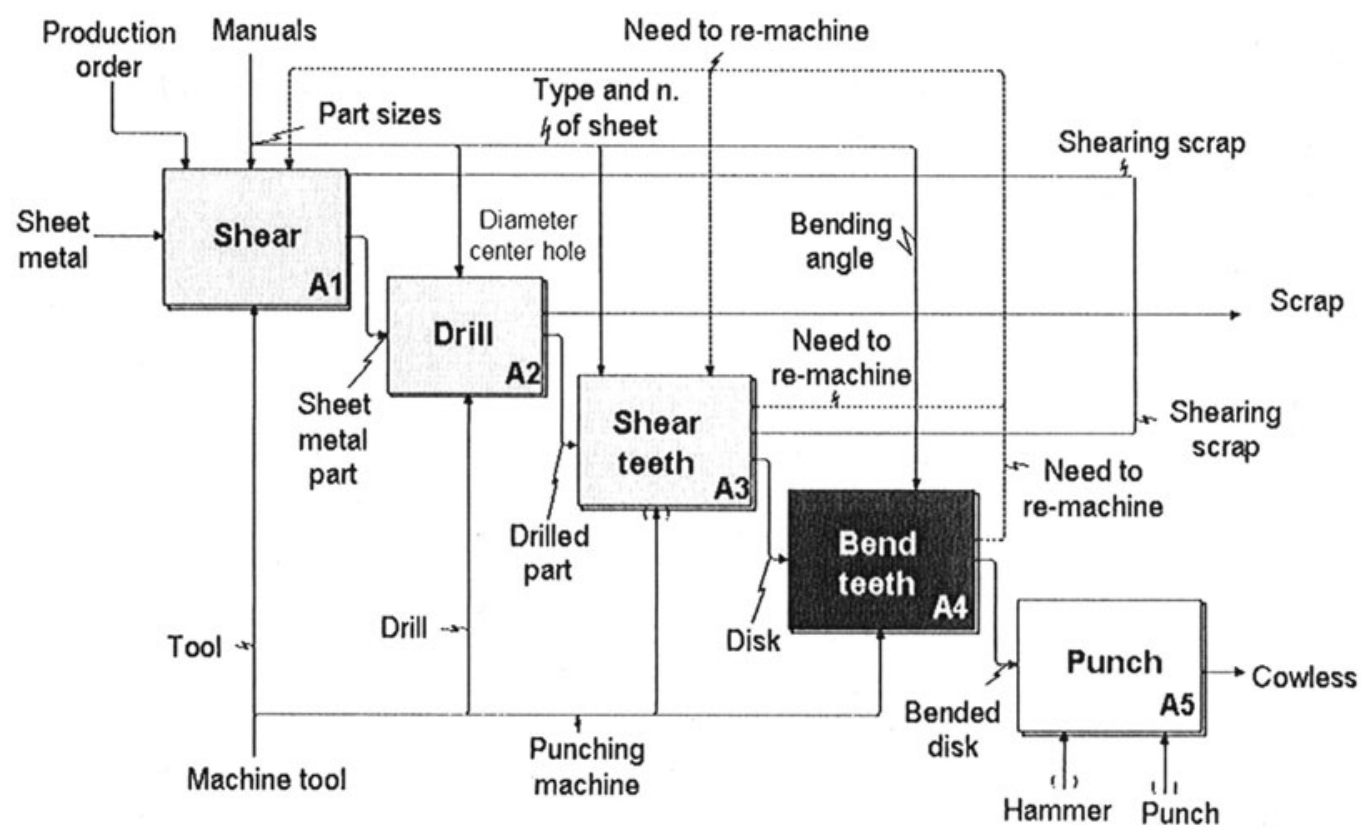

Figure 4. A0 Diagram: Produce Cowless

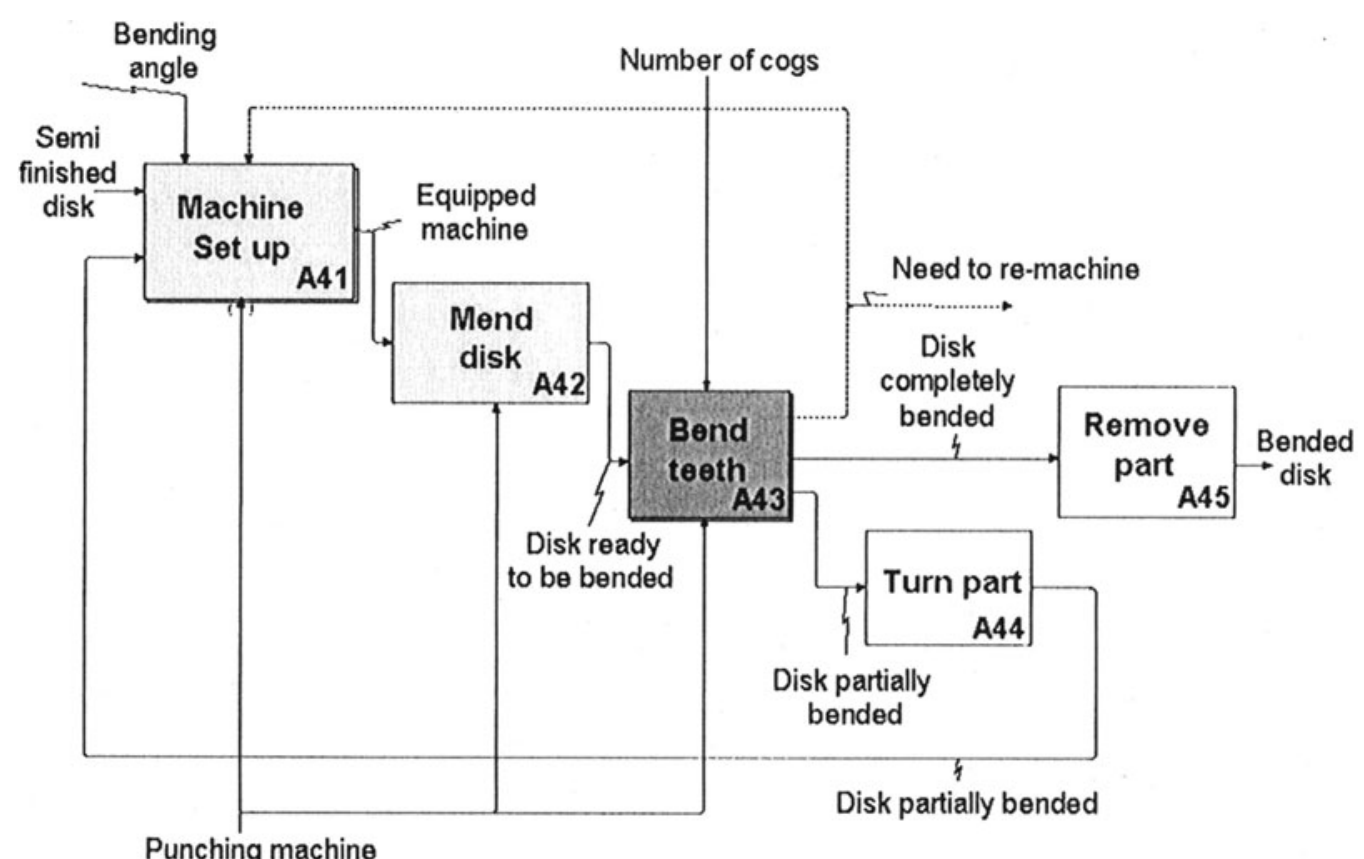

Figure 5. A4 Diagram: Bend teeth

This phase was carried out in teamwork with company's technical staff, and the final result has been a deep knowledge of both AS- IS processes, graphically represented and described in a structured documentation. In 
particular, process documentation comprehends a set of IDEF0 diagrams, hierarchically organized, and a glossary to explain terminology used.

\subsection{Problem solving}

Second step consisted in analyzing and solving the problems using the TRIZ methodology with the support of TechOptimizer by Invention Corp. The strategy adopted is schematized in Figure 6.
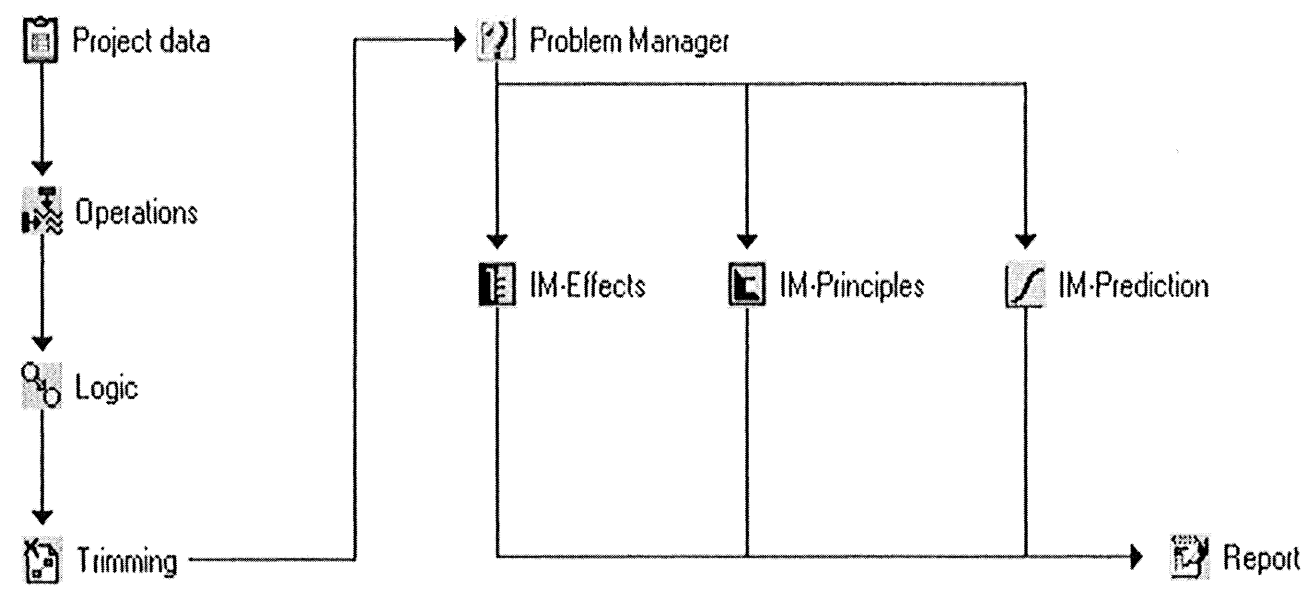

Figure 6. Adopted solving strategy (by TechOptimizer)

First project data were defined: objectives, limitations, parameters to be improved and the importance of each one. The parameters chosen are reported in Table 1.

Table 1. Project parameters

\begin{tabular}{|l|l|l|l|}
\hline Parameter & Symbol & Condition & Importance \\
\hline Function rank & $\mathrm{F}$ & $\mathrm{Up}$ & 6 \\
\hline Problem rank & $\mathrm{P}$ & Down & 8 \\
\hline Cost & $\mathrm{C}$ & Down & 8 \\
\hline Noise & $\mathrm{N}$ & Down & 6 \\
\hline Labor expenditure & LE & Down & 10 \\
\hline Losses of product material & LoP & Down & 1 \\
\hline Mistakes & $\mathrm{Mi}$ & Down & 6 \\
\hline
\end{tabular}

Then, on the basis of AS-IS process analysis, functional models of both the product and the process have been realized in order to identify respectively critical components and operations. The product model shows all the interactions between the cowless and the components of the production process. Every component was valued according to the chosen 
parameters (Table 1) in order to find out the less efficient. Typically, TechOptimizer evaluates all the interactions between the parts of the object, makes a set of calculations, and makes a diagnosis: what parts of the system should be improved, how the system should be simplified, etc. In our case, the analysis demonstrated that was necessary to reduce human intervention implementing a more automated process.

The process model represents all single operations, their precedences and logical links. Figure 7 portrays a part of process model for cowless teeth bending. According to system philosophy, each operation was classified into productive (Prd), providing (Prv), harmful $(\mathrm{H})$ or corrective $(\mathrm{Co})$ and, as the component of the product model, was valued with respect to the chosen parameters (Table 1).

The applied strategy is based on trimming concept by which a process can be simplified by eliminating critical or insufficient operations of the process, while redistributing the operation's useful functions to other operations or to non-processed components. Redistribution of functions means fewer and simpler operations, and by eliminating operations, there are fewer problems to be solved (TechOptimizer 1999). Therefore, trimming is a tool for eliminating or simplifying an operation of the process while retaining or transferring its useful functions. To do this, TechOptimizer uses a component evaluation algorithm to choose the order in which to trim elements.

In our application many trimmings were operated on the process model in order to remove insufficient operations (e.g., Figure 7 - To Bend 1 operation). Useful functions of the trimmed operations have been transferred to remaining ones or to new operations that have been introduced (Figure 8).

Initial problems are so expressed in different ways that can be approached with the solving modules of the package.

Eight trimming variants were proposed to investigate different scenarios:

- Three consider a unique machine performing both shear and bending. They represent the most advanced solutions but also the most difficult and expensive to implement.

- Four consider two different machines: one for shear operation and another one for the bending.

- The last one is an intermediate solution; it considers a new machine for shear and partial bending, and the existing machine to complete the task.

Once performed trimming, to find out solutions for the eight variants we used the solving modules of TechOptimizer, based on TRIZ theory (TechOptimizer 1999): Prediction, Principles, and Effects. 


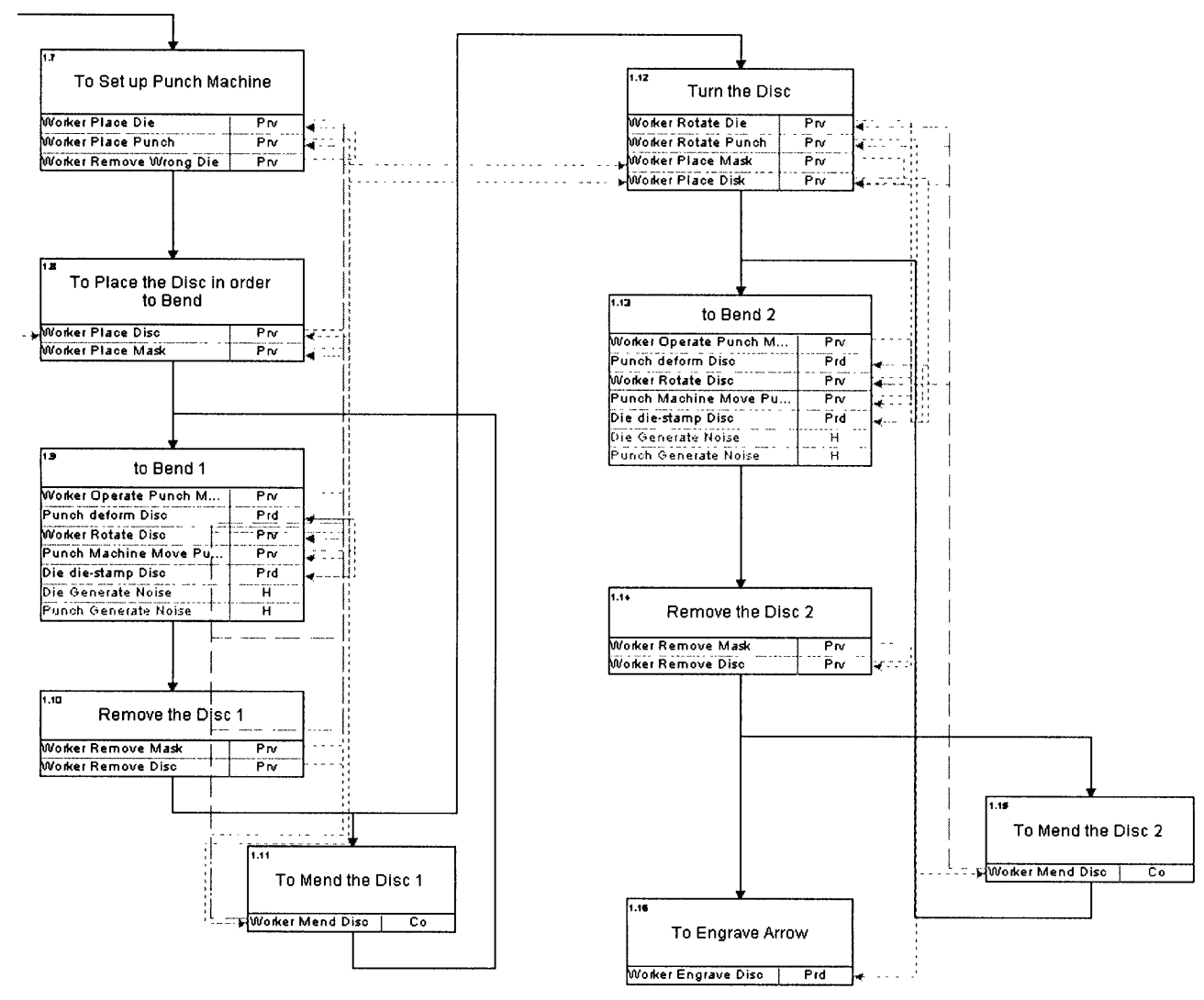

Figure 7. A shot of Functional model for cowless production process

\begin{tabular}{|l|l|l|}
\hline \multicolumn{1}{|c|}{ to Bend1 } \\
\hline Wronker Operate Punch Machine \\
\hline Punch deform Disc \\
\hline Wonker Rotate Disc \\
\hline Punch Machine Move Punch \\
\hline Die die-stamp Disc \\
\hline Die Generate Noise \\
\hline Punch Generate Noise
\end{tabular}

Figure 8. Trim of to Bend1 operation and transferring to a new operation

Prediction tool solves technical problems involving interaction between objects. This module identifies key evolutionary trends of technology and clearly predicts future directions of scientific innovation.

With Prediction tool, considering the statement that "there are standard trends of evolution that every technical system follow in its development", 
we found that mechanical interactions will be progressively reduced and even eliminated. This statement suggested a solution for cutting problem (Figure 9) that can be also considered for bending operation.

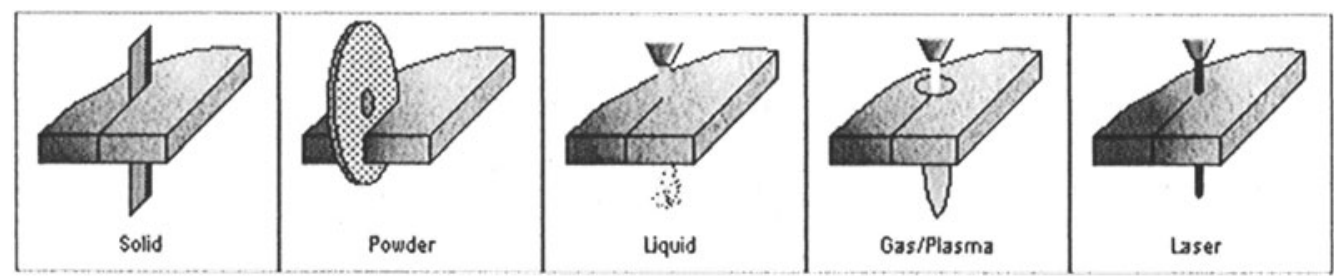

Figure 9. Prediction tool (by TechOptimizer)

Principles tool shows solutions to engineering contradictions. This module allows users to produce optimal solutions by introducing the best inventive principles applicable for a specific scientific problem. This module is based on the evaluation of over 3 million patents across many fields of engineering.

The Principles tool suggested restating the problem as a technical contradiction between improving and worsening parameters. In our case there was a contradiction between a reduction of operation time and an increase of the device/machine complexity. This contradiction can be solved with the principle of multifunctionality, i.e. using a component to perform several different functions, for example the punch.

Another technical contradiction was increase of versatility and increase of machine complexity. It can be solved with the principle of changing state. For example for bending operation we can soften bending lines by heating them, or using physical fields in place of mechanical devices, as already found with Prediction tool.

The last module, called Effects, allows the user to access over 4,400 engineering and scientific effects. It provides a database of theorems, laws and phenomena that permits to look for solution to similar problems. For example, an effect suggested implementing the principle of changing state with a laser beam, another one with electromagnetic forming. Other effects suggested for cutting are advanced method of high-pressure water jet cutting and gas-oxygen cutting of metal.

\subsection{Modeling and Simulation of the TO-BE Processes}

Among the eight possible configurations, i.e., eight possible TO-BE processes, we selected, in collaboration with the technical staff of the company, two of them. 
The first is more conservative and easy to implement. It considers two distinct machines: one for water jet cutting and another one for bending with a double punch (Principle Multifunctionality).

The latter is more innovative and requires the development and installation of only one machine to produce the component. It is based on the use of laser technology for cutting and electromagnetic forming for bending. This solution has been mainly considered for future development.

Two TO-BE processes have been modeled and simulated. Figure 10 and 11 show the IDEF3 models respectively for the solution with two machines and for that one with one machine.

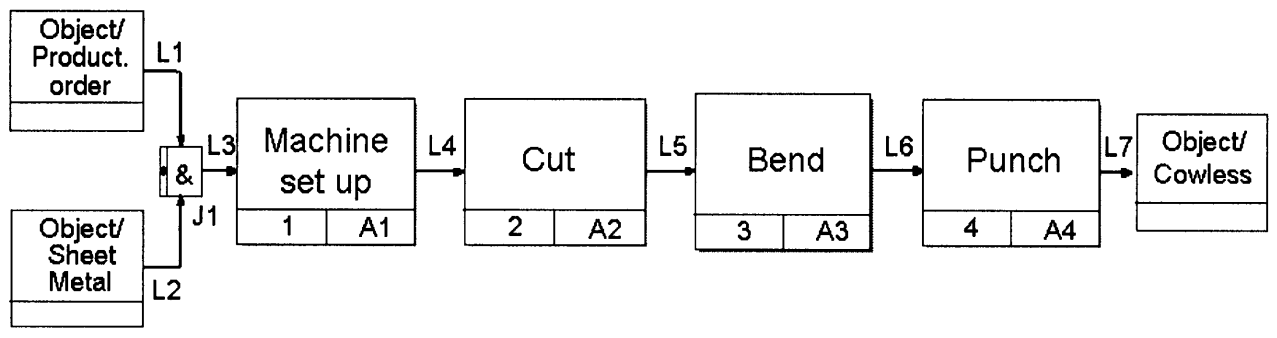

Figure 10. IDEF3 model: two distinct machines for cutting and bending

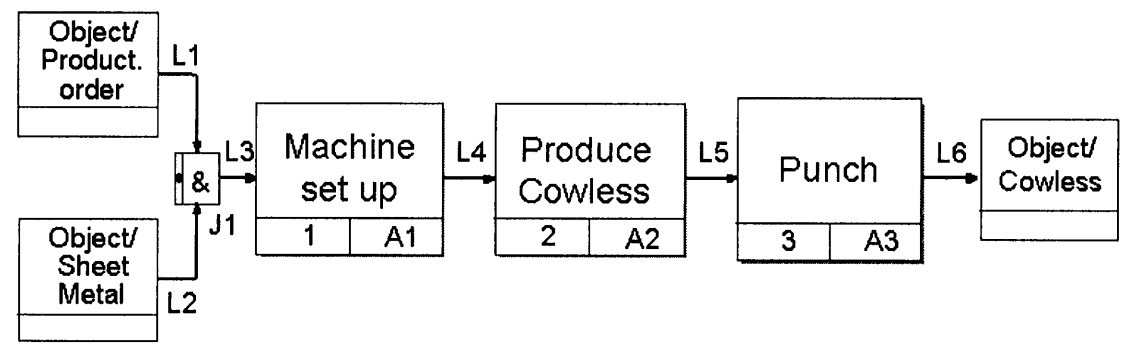

Figure 11. IDEF3 model: a unique machines for cutting and bending

The simulation of the TO-BE processes has been carried out with respect to production time and resources considering different typologies of the component and different lot size. Simulation data obtained have been compared with those obtained during first phase. Figure 12 and 13 portray the comparison among AS-IS process and the TO-BE processes respectively for production time and manpower.

The new configurations show a good reduction both for production time and manpower. Best results are for small lot size; in fact the mail goal of 
adopted re-engineering strategy has been to reduce time for machine set-up in order to increase process flexibility.

Anyway, data concerning the second solution (a unique machine for cutting and bending) has to be considered indicative, because, even if we have precise data regarding laser cutting (used by sub-contractors) the technology proposed for bending (electromagnetic forming) requires further investigation.

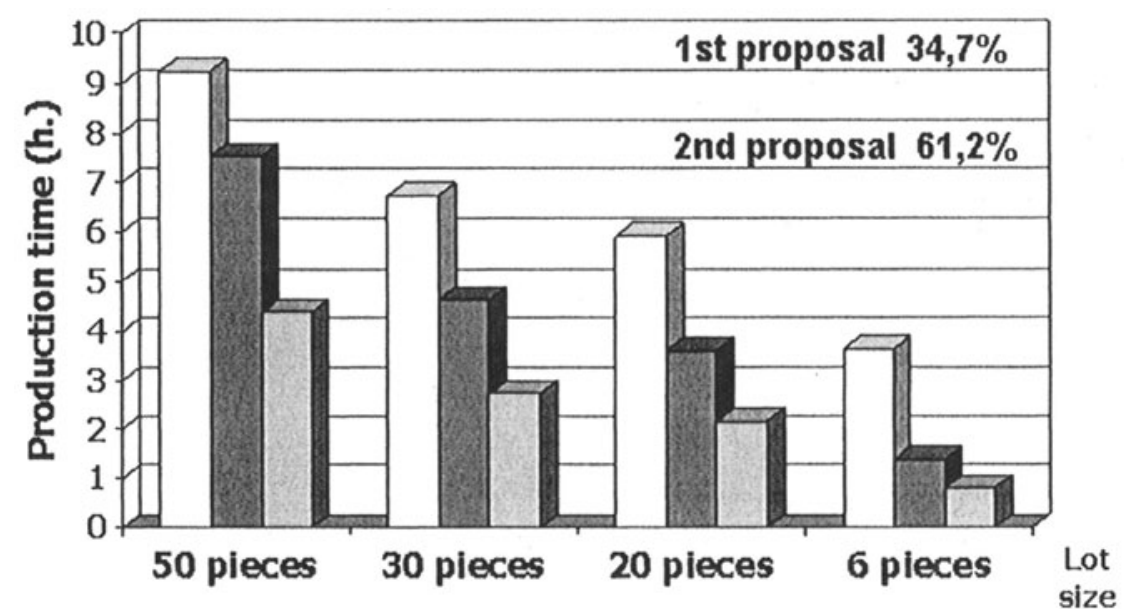

$\square$ AS-IS (1st Proposal $\square$ 2nd Proposal

Figure 12. Production time

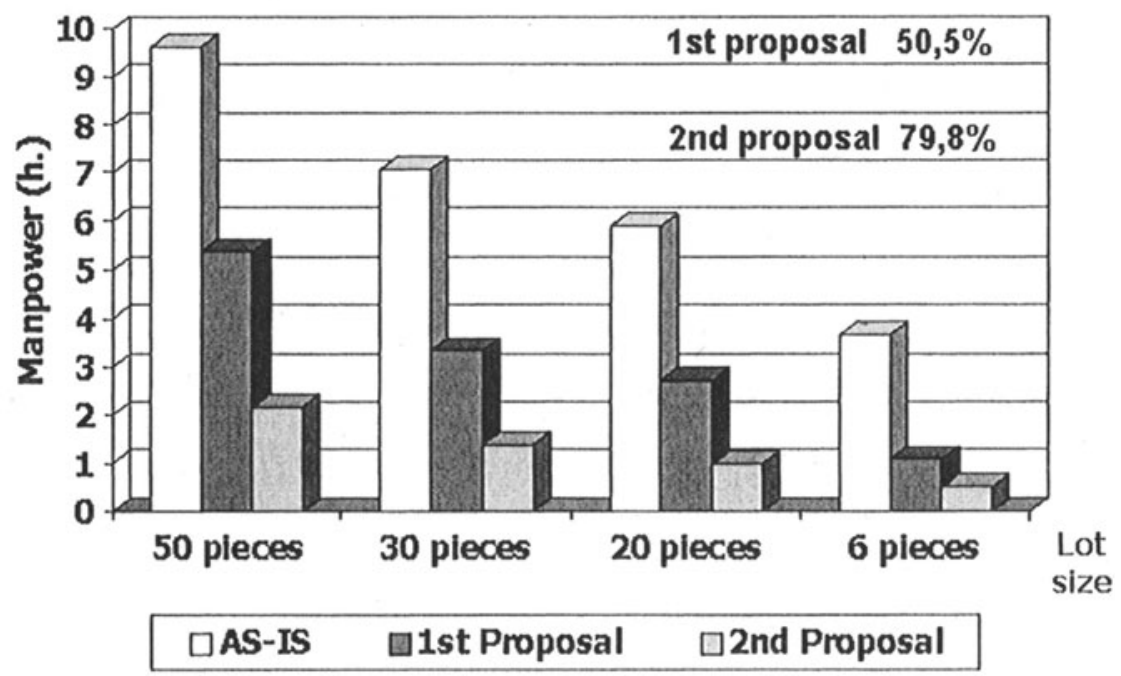

Figure 13. Manpower 


\section{CONCLUSIONS}

In this paper we propose a methodology to acquire and formalize process knowledge for technological innovation and for Business Process Reengineering. It is based on the integration of process modeling and simulation techniques with TRIZ Theory. The proposed set of tools and the road map have been experimented and validated with an industrial test case: the production process of a mixer component. In particular, recognizable benefits of the proposed approach are:

- Definition of a new reference road map for process re-engineering based on the integration of IDEF and business process simulation techniques with TRIZ theory;

- Possibility to define new production processes and to evaluate them quantitatively thanks to adopted techniques and tools; in fact, they permit to measure and report on productivity and innovation with respect to reengineering objectives;

- Production of structured process documentation that formalizes AS-IS and TO-BE process knowledge and permits to spread and share knowledge among company departments;

- Possibility to share our expertise and promote a fruitful exchange of opinions and experience with process expert of the company.

Summarizing, modeling and simulation techniques permit to capture and formalize the knowledge related product development process, making it available to the different process actors (from designer to shop-floor operator) and improving product and process data/knowledge sharing.

Finally, following future developments have been considered:

- From company point of view: to perform further investigation on the techniques based on electromagnetic forming for bending operation;

- From academic point of view: to investigate new frameworks for engineering design processes (Salustri 2000) and to verify to what extent it could be possible to map automatically IDEF models into structural functional model generated with TechOptimizer.

\section{REFERENCES}

Altshuller G., And Suddenly the Inventor Appeared.TRIZ. The Theory of Inventive Solving Problems, Technical Innovation Center, Inc., Worcester, Massachuttes, 1996.

Altshuller G., "Knowledge-based Innovation - a Technology of the Future". In From Knowledge Intensive CAD to Knowledge Intensive Engineering, Eds U. Cugini \& M. Wozny, Kluwer Academic Publishers, 2001.

Bozza, I., Cugini, U., Ruozi, D., and Petrotta, G. "Modeling and Simulating Improvements of the Ferrari Auto Steering Gear Development Process". Proceedings of 31st ISATA 
International Symposium on AutomotiveTechnology \& Automation, Advanced Manufacturing/Innovation Management, ISATA, 1998, Croydon, UK.

Lawton G. Industry Trends - Knowledge Management: Ready for Prime Time? Computer, February 2001, IEEE Computer Society.

Retrieved from: http://www.computer.org

Mayer R.J., deWitte P.S. Delivering Results: Evolving BPR from Art to Engineering. Retrieved March 2002 from: http://www.idef.com/Downloads/free downloads.html (to be published in a forthcoming book on Business Process Reengineering by Kluwer).

Nakahawa T. Let's learn TRIZ. 1999

Retrieved from: http://www.osaka-gu.ac.jp/php/nakagawa/TRIZ.

Nomaguchi Y., Yoshioka M., and Tomiyama T. "Document-based Design Process Knowledge Management for Knowledge Intensive Engineering". In From Knowledge Intensive CAD to Knowledge Intensive Engineering, Eds U. Cugini \& M. Wozny, Kluwer Academic Publishers, 2001.

Salustri F. "Towards a logical Framework for engineering design processes". In From Knowledge Intensive CAD to Knowledge Intensive Engineering, Eds U. Cugini \& M. Wozny, Kluwer Academic Publishers, 2001.

Scarbrough H. (1996). Business Process Re-design: the Knowledge Dimension.

Retrieved from: http://bprc.warwick.ac.uk/rc-rep-8.html.

TechOptimizer. Reference Manual. Invention Machine Corporation, 1999. 